\title{
A second order convergent trial method for a free boundary problem in three dimensions
}

\author{
Monica Bugeanu \\ Department for Mathematics and Computer Science, Spiegelgasse 1, 4051 Basel, Switzerland \\ E-mail:monica.bugeanu@unibas.ch \\ HELMUT HARBRECHT \\ Department for Mathematics and Computer Science, Spiegelgasse 1, 4051 Basel, Switzerland \\ E-mail: helmut.harbrecht@unibas.ch
}

[Received 26 September 2014 and in revised form 27 August 2015]

\begin{abstract}
The present article is concerned with the solution of a generalized Bernoulli free boundary problem in three spatial dimensions. We parametrize the free boundary under consideration over the sphere and apply a trial method which updates the free boundary into the normal direction. At the free boundary, we prescribe the Neumann boundary condition and update the free boundary with the help of the remaining Dirichlet boundary condition. An inexact Newton update is employed which leads to a novel second order convergent trial method. Numerical examples show the feasibility of the present approach. In particular, a parametric representation is utilized which imposes no restriction to the free boundary under consideration except for its genus.
\end{abstract}

2010 Mathematics Subject Classification: Primary 65N99; Secondary 35R35.

Keywords: Free boundary problem; trial method; second order convergence.

\section{Introduction}

The present article is devoted to the numerical solution of a free boundary problem of Bernoulli type by a second order convergent trial method. Let $S \subset \mathbb{R}^{3}$ denote a bounded and simply connected domain with boundary $\Sigma:=\partial S$. Moreover, assume a second simply connected domain $T \subset \mathbb{R}^{3}$ with free boundary $\Gamma:=\partial T$ which contains the first domain, i.e. $\bar{S} \subset T$. The resulting annular domain is denoted by $\Omega:=T \backslash \bar{S}$, see also Figure 1 for an illustration.

The free boundary problem now consists in finding a domain $\Omega$ and an associated function $u=u(\Omega) \in H^{1}(\Omega)$ such that the following overdetermined problem is satisfied:

$$
\begin{aligned}
-\Delta u=f & \text { in } \Omega, \\
u=g & \text { on } \Sigma, \\
-\frac{\partial u}{\partial \mathbf{n}}=h, \quad u=0 & \text { on } \Gamma .
\end{aligned}
$$

Throughout this article, we will assume that $g, h>0$ and $f \geqslant 0$ are sufficiently smooth functions on $\mathbb{R}^{3}$ such that $u \in C^{2}(\bar{\Omega} \cap U)$ with $U$ being a tubular neighbourhood of the free boundary $\Gamma$. We like to stress that the positivity of the Dirichlet data at the interior boundary implies (due to the maximum principle and the unique continuation property [23]) that $u$ is positive on $\Omega$ and thus in fact $\partial u / \partial \mathbf{n}<0$. 


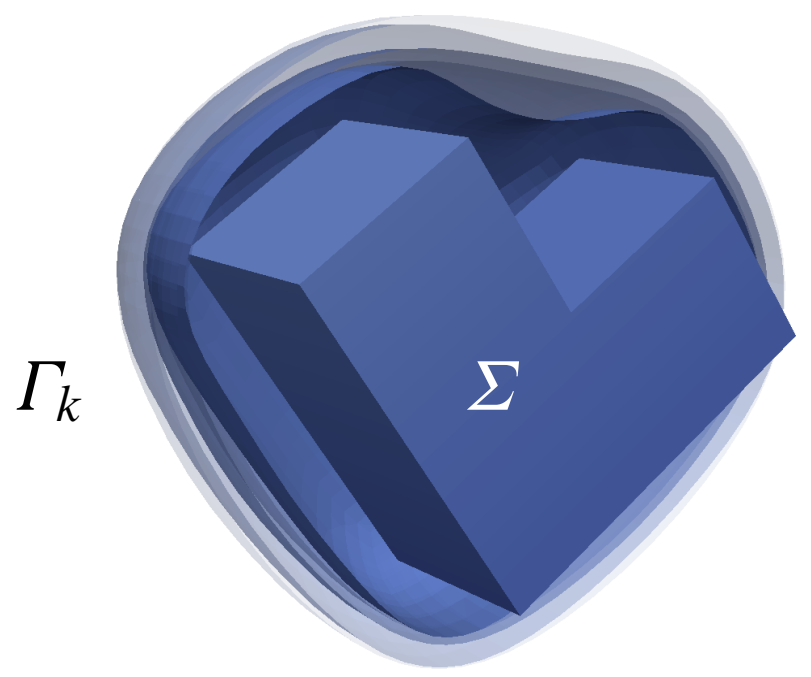

FIG. 1. The interior boundary $\Sigma$ and different approximations $\Gamma_{k}$ to the sought free boundary $\Gamma$

The trial method is a fixed point iteration for the sought free boundary. For the current free boundary, the solution of (1.1) is computed with one boundary condition at the free boundary and the other boundary condition is used for updating the free boundary. Trial methods for Bernoulli's free boundary problem based on a parametrization of the free boundary have been proposed in, e.g., $[1,12,35,36]$. Whereas, the level set method for representing the free boundary has been used in $[4,5,26]$, enjoying the property of allowing topology changes.

An alternative approach for solving free boundary problems is shape optimization, see, e.g., [8, 9, 20, 21, 24, 32] and the references therein. In particular, in [15] a Newton method has been proposed for three dimensional free boundary problems. The different reformulations of Bernoulli type free boundary problems as shape optimization problems have been compared in [11]. We should finally also mention the pseudo-solid approach as a further solution technique for computing the solution of free boundary problems, see [22, 30, 37] for example.

The basic idea of the trial method which we propose in this article has been introduced and analyzed in [16]. There, a trial method has been proposed whose convergence towards the optimal solution is locally quadratic. Nevertheless, for sake of simplicity, we restricted ourselves to twodimensional domains which are starlike and updated the free boundary only in the radial direction. Contrary to this, we consider now arbitrary three-dimensional domains of genus zero which are updated in the normal direction. This makes the trial method and the analysis different. In addition, the algorithmic treatment is much more complicated in three spatial dimensions.

The proposed trial method imposes the Neumann boundary condition at the free boundary and updates the free boundary according to the Dirichlet boundary condition. The particular update corresponds to an inexact Newton update and involves the so-called local shape derivative. The latter is a measure for the sensitivity when deforming the current computational domain by a given perturbation field. It is a well known quantity in shape calculus, see, e.g., [8, 27, 31, 32] and the references therein. 
We like to mention that a second order convergent trial method has already been proposed many years ago in $[12,36]$. It is based on a Robin boundary value problem which involves the mean curvature of the free boundary. Unfortunately, if the free boundary becomes non-convex, this boundary value problem is not elliptic any more and the trial method might diverge. This serious drawback is avoided by the trial method used in this article.

Throughout this article, we will not consider the interesting question of existence of optimal solutions. Instead, we will tacitly assume the existence of optimal domains, being sufficiently regular to apply shape calculus. For the existence of solutions to the generalized exterior Bernoulli free boundary problem (1.1), we refer the reader to, e.g., [3], see also [12] for the related interior free boundary problem. Results concerning the geometric form of the solutions can be found in [2] and the references therein.

The outline of this article is as follows. Section 2 is dedicated to the trial method under consideration. The discretization of the free boundary is the topic of Section 3. Then, in Section 4, we discuss the efficient computation of the state by means of a fast wavelet based boundary element method. In Section 5, we perform numerical experiments to demonstrate the feasibility of the trial method. Finally, in Section 6, we state concluding remarks.

\section{Trial method}

\subsection{Background and motivation}

Throughout this article, we shall assume that the free boundary is of genus zero. Then, we can identify the domain $\Omega$ with a parametrization $\gamma: \mathbb{S} \rightarrow \Gamma$ of its free boundary $\Gamma$. Here, $\mathbb{S}=\{\mathbf{x} \in$ $\mathbb{R}^{3}:\|\mathbf{x}\|=1$ \} denotes the three-dimensional unit sphere.

The trial method for the solution of the free boundary problem (1.1) is a fixed point method for the unknown parametrization $\boldsymbol{\gamma}$ which reads as follows:

(1) Choose an initial guess $\Gamma_{0}$ of the free boundary and set $k=0$.

(2a) Solve the boundary value problem

$$
\begin{aligned}
-\Delta u_{k}=f & & \text { in } \Omega_{k}, \\
u_{k}=g & & \text { on } \Sigma, \\
-\frac{\partial u_{k}}{\partial \mathbf{n}_{k}}=h & & \text { on } \Gamma_{k} .
\end{aligned}
$$

(2b) Update the free boundary $\Gamma_{k}$ by moving the old boundary into the normal direction $\mathbf{n}_{k}$ :

$$
\boldsymbol{\gamma}_{k+1}=\boldsymbol{\gamma}_{k}+\delta_{k} \mathbf{n}_{k}
$$

(3) Increase $k \mapsto k+1$ and repeat step (2) until the process becomes stationary up to a specified accuracy.

The most common update rule $\delta_{k}^{(\text {std) }}=\Psi\left(\gamma_{k}\right) \in C^{2}(\mathbb{S})$ for calculating the update in (2.2) is determined in such a way that the desired homogeneous Dirichlet boundary condition is satisfied at the new boundary $\Gamma_{k+1}$. This means that, given $u_{k}$ which satisfies (2.1), one makes the ansatz

$$
u_{k} \circ \gamma_{k+1} \stackrel{!}{=} 0 \quad \text { on } \mathbb{S},
$$


where $u_{k}$ is assumed to be smoothly extended into the exterior of $\Omega_{k}$. The desired update is found by linearizing $u_{k} \circ \gamma_{k+1}=u_{k} \circ\left(\gamma_{k}+\delta_{k} \mathbf{n}_{k}\right)$ with respect to the update function $\delta_{k}$. This yields the equation

$$
0=u_{k} \circ \boldsymbol{\gamma}_{k+1} \approx u_{k} \circ \boldsymbol{\gamma}_{k}+\left(\frac{\partial u_{k}}{\partial \mathbf{n}_{k}} \circ \boldsymbol{\gamma}_{k}\right) \delta_{k} .
$$

Inserting the Neumann boundary condition $-\partial u_{k} / \partial \mathbf{n}_{k}=h$ and solving for $\delta_{k}$, we arrive at the following condition for the standard update:

$$
\delta_{k}^{(\mathrm{std})}=\Psi\left(\gamma_{k}\right):=\frac{u_{k} \circ \gamma_{k}}{h \circ \gamma_{k}}: \mathbb{S} \rightarrow \mathbb{R}
$$

This update rule has been used in, e.g., $[12,26,36]$ and yields a first order convergent trial method (see [36]). Notice that (2.5) is well defined since we assumed $h>0$ to ensure the solvability of the free boundary problem.

REMARK 2.1 The update $\delta_{k}^{(\text {std) }}$ from (2.5) is derived from the first order Taylor expansion (2.4). Instead, a second order Taylor expansion can also be used which would lead to a more stable trial method that, however, is also only first order convergent, cf. [16] for the details.

\subsection{Shape sensitivity analysis}

For sake of convenience, we skip the iteration index $k$ and denote the free boundary just by $\Gamma$. The associated annular domain is denoted by $\Omega$. Given a sufficiently smooth boundary perturbation field $\mathbf{V}: \Gamma \rightarrow \mathbb{R}^{3}$, we can define the perturbed boundary $\Gamma_{\varepsilon}=\Gamma_{\varepsilon}[\mathbf{V}]$ by

$$
\Gamma_{\varepsilon}:=\{(\mathbf{I}+\varepsilon \mathbf{V})(\mathbf{x}): \mathbf{x} \in \Gamma\} .
$$

This boundary defines the perturbed domain $\Omega_{\varepsilon}$.

Let $u$ and $u_{\varepsilon}$ denote the solutions of (2.1) with respect to the domains $\Omega$ and $\Omega_{\varepsilon}$, i.e.,

$$
\begin{aligned}
& -\Delta u=f \quad \text { in } \Omega, \quad-\Delta u_{\varepsilon}=f \quad \text { in } \Omega_{\varepsilon}, \\
& \begin{array}{llll}
u=g & \text { on } \Sigma, & u_{\varepsilon}=g & \text { on } \Sigma,
\end{array} \\
& -\frac{\partial u}{\partial \mathbf{n}}=h \quad \text { on } \Gamma, \quad-\frac{\partial u_{\varepsilon}}{\partial \mathbf{n}}=h \quad \text { on } \Gamma_{\varepsilon} .
\end{aligned}
$$

Then, the local shape derivative $\mathrm{d} u=\mathrm{d} u[\mathbf{V}]$ of $u$ at $\Omega$ in the direction $\mathbf{V}$ is formally (see [27, 32] for a rigorous derivation) obtained by the pointwise limit

$$
\mathrm{d} u(\mathbf{x})=\lim _{\varepsilon \rightarrow 0} \frac{u_{\varepsilon}(\mathbf{x})-u(\mathbf{x})}{\varepsilon}, \quad \mathbf{x} \in \Omega \cap \Omega_{\varepsilon} .
$$

The local shape derivative measures the sensitivity of the solution to (2.1) when changing the domain $\Omega$ in the direction $\mathbf{V}$.

In accordance with $[8,32]$, the local shape derivative can be characterized by a boundary value problem. 
LEMmA 2.2 Given a sufficiently smooth boundary perturbation field $\mathbf{V}: \Gamma \rightarrow \mathbb{R}^{3}$. Then, the local shape derivative $\mathrm{d} u=\mathrm{d} u[\mathbf{V}]$ of the boundary value problem (2.1) is given as the solution of the problem

$$
\begin{aligned}
\Delta \mathrm{d} u & =0 & & \text { in } \Omega \\
\mathrm{d} u & =0 & & \text { on } \Sigma \\
\frac{\partial \mathrm{d} u}{\partial \mathbf{n}} & =\left[f-2 \nVdash h-\frac{\partial h}{\partial \mathbf{n}}\right]\langle\mathbf{V}, \mathbf{n}\rangle+\operatorname{div}_{\Gamma}\left(\langle\mathbf{V}, \mathbf{n}\rangle \nabla_{\Gamma} u\right) & & \text { on } \Gamma .
\end{aligned}
$$

Note that the operator $\operatorname{div}_{\Gamma}$ denotes the surface divergence, the operator $\nabla_{\Gamma}$ denotes the surface gradient, and $\mathcal{H}$ denotes the mean curvature of the surface $\Gamma$. For more details concerning shape sensitivity analysis, we address the reader to $[8,27,28,32]$.

\subsection{Speeding up the convergence}

We shall go back to the trial method (2.2) and the update rule (2.5). Clearly, this trial method converges if $\delta_{k}^{\text {(std) }}=\Psi\left(\gamma_{k}\right) \rightarrow 0$ as $k \rightarrow \infty$. Hence, in order to arrive at a second order convergent trial method, we intend to directly solve the equation

$$
\Psi\left(\gamma_{k}\right) \stackrel{!}{=} 0 \quad \text { on } \mathbb{S}
$$

by a Newton-type method. To that end, we prove first the following theorem.

THEOREM 2.3 The shape derivative of the update function

$$
\Psi(\gamma):=\frac{u \circ \gamma}{h \circ \gamma} .
$$

in the direction $\mathbf{V}=\delta \mathbf{n}$ is given by

$$
\frac{\partial \Psi}{\partial(\delta \mathbf{n})}(\boldsymbol{\gamma})=-\delta-\delta \frac{u \circ \boldsymbol{\gamma}}{(h \circ \boldsymbol{\gamma})^{2}}\left(\frac{\partial h}{\partial \mathbf{n}} \circ \boldsymbol{\gamma}\right)+\frac{\mathrm{d} u[\delta \mathbf{n}] \circ \boldsymbol{\gamma}}{h \circ \boldsymbol{\gamma}},
$$

where $\mathrm{d} u=\mathrm{d} u[\delta \mathbf{n}]$ is the local shape derivative (2.7) in the direction $\mathbf{V}=\delta \mathbf{n}$.

Proof. Let the actual boundary $\Gamma$ be described by $\gamma$ and let the perturbed boundary be described by $\boldsymbol{\gamma}_{\varepsilon}=\boldsymbol{\gamma}+\varepsilon \delta \mathbf{n}$. Let $u$ and $u_{\varepsilon}$ denote the solutions to the underlying boundary value problems (2.6) relative to the domains $\Omega$ and $\Omega_{\varepsilon}$. Then, we have

$$
\begin{aligned}
\frac{\partial \Psi}{\partial(\delta \mathbf{n})}(\boldsymbol{\gamma}) & =\lim _{\varepsilon \rightarrow 0} \frac{\Psi\left(\boldsymbol{\gamma}_{\varepsilon}\right)-\Psi(\boldsymbol{\gamma})}{\varepsilon} \\
& =\lim _{\varepsilon \rightarrow 0} \frac{1}{\varepsilon}\left(\frac{u_{\varepsilon} \circ \gamma_{\varepsilon}}{h \circ \gamma_{\varepsilon}}-\frac{u \circ \boldsymbol{\gamma}}{h \circ \gamma}\right) \\
& =\lim _{\varepsilon \rightarrow 0} \frac{1}{\varepsilon}\left(\left[\frac{u_{\varepsilon} \circ \gamma_{\varepsilon}}{h \circ \gamma_{\varepsilon}}-\frac{u_{\varepsilon} \circ \gamma}{h \circ \gamma_{\varepsilon}}\right]+\left[\frac{u_{\varepsilon} \circ \gamma}{h \circ \gamma_{\varepsilon}}-\frac{u_{\varepsilon} \circ \gamma}{h \circ \gamma}\right]+\left[\frac{u_{\varepsilon} \circ \gamma}{h \circ \gamma}-\frac{u \circ \gamma}{h \circ \gamma}\right]\right) \\
& =A+B+C .
\end{aligned}
$$

Computing the limit of the first term on the right hand side gives

$$
A=\lim _{\varepsilon \rightarrow 0} \frac{1}{\varepsilon} \frac{u_{\varepsilon} \circ \boldsymbol{\gamma}_{\varepsilon}-u_{\varepsilon} \circ \boldsymbol{\gamma}}{h \circ \boldsymbol{\gamma}_{\varepsilon}}=\frac{\langle\nabla u, \delta \mathbf{n}\rangle}{h \circ \boldsymbol{\gamma}}=\delta \frac{\frac{\partial u}{\partial \mathbf{n}} \circ \boldsymbol{\gamma}}{h \circ \boldsymbol{\gamma}} .
$$


By inserting the Neumann boundary condition $-\partial u / \partial \mathbf{n}=h$, we arrive at $A=-\delta$. Next, we compute the limit of the second term on the right hand side:

$$
B=\lim _{\varepsilon \rightarrow 0} \frac{1}{\varepsilon}\left(\frac{u_{\varepsilon} \circ \boldsymbol{\gamma}}{h \circ \gamma_{\varepsilon}}-\frac{u_{\varepsilon} \circ \boldsymbol{\gamma}}{h \circ \boldsymbol{\gamma}}\right)=-\frac{u \circ \boldsymbol{\gamma}}{(h \circ \boldsymbol{\gamma})^{2}}\langle\nabla h, \delta \mathbf{n}\rangle=-\delta \frac{u \circ \boldsymbol{\gamma}}{(h \circ \boldsymbol{\gamma})^{2}}\left(\frac{\partial h}{\partial \mathbf{n}} \circ \boldsymbol{\gamma}\right) .
$$

Finally, with the help of the local shape derivative, the limit of the last term on the right hand side is given by

$$
C=\lim _{\varepsilon \rightarrow 0} \frac{1}{\varepsilon} \frac{u_{\varepsilon} \circ \boldsymbol{\gamma}-u \circ \boldsymbol{\gamma}}{h \circ \boldsymbol{\gamma}}=\lim _{\varepsilon \rightarrow 0} \frac{1}{\varepsilon} \frac{\left(u_{\varepsilon}-u\right) \circ \boldsymbol{\gamma}}{h \circ \boldsymbol{\gamma}}=\frac{\mathrm{d} u[\delta \mathbf{n}] \circ \boldsymbol{\gamma}}{h \circ \boldsymbol{\gamma}},
$$

see, e.g., $[8,32]$ for the details. By putting all together, we arrive at the desired derivative (2.9).

As a consequence of this theorem, the Newton method can be applied to solve the equation (2.8). Nevertheless, in view of the Dirichlet boundary condition $u=0$ at the sought free boundary, the second term in the shape derivative (2.9) of the update function vanishes at the optimum boundary. Hence, we can simplify the Newton method by determining the inexact Newton update $\delta_{k}^{(\mathrm{scd})}$ which is implicitly given by

$$
\delta_{k}^{(\mathrm{scd})}-\frac{\mathrm{d} u_{k}\left[\delta_{k}^{(\mathrm{scd})} \mathbf{n}_{k}\right] \circ \boldsymbol{\gamma}_{k}}{h \circ \boldsymbol{\gamma}_{k}} \stackrel{!}{=} \Psi\left(\boldsymbol{\gamma}_{k}\right) .
$$

Since the neglected term is of second order in the size of the desired update, the resulting inexact Newton method will also converge (locally) quadratically to the sought free boundary, see, e.g., [13]. Nevertheless, we emphasize that the update (2.10) coincides with the Newton update if the field $h$ is constant. This holds for example in case of Bernoulli's original free boundary problem.

\section{Discretization of the free boundary}

\subsection{Finite dimensional surface representation}

We shall first introduce the spherical harmonics. For $n \in \mathbb{N}_{0}$ and $|m| \leqslant n$, consider the Legendre polynomials

$$
P_{n}(t):=\frac{1}{2^{n} n !}\left(\frac{\mathrm{d}}{\mathrm{d} t}\right)^{n}\left(t^{2}-1\right)^{n}, \quad t \in \mathbb{R},
$$

and the associated Legendre functions

$$
P_{n}^{|m|}(t):=\left(1-t^{2}\right)^{|m| / 2}\left(\frac{\mathrm{d}}{\mathrm{d} t}\right)^{|m|} P_{n}(t), \quad t \in \mathbb{R} .
$$

Then, the spherical harmonics $Y_{n}^{m}: \mathbb{S} \rightarrow \mathbb{R}$ are given by

$$
Y_{n}^{m}(\widehat{\mathbf{x}}):=\sqrt{\frac{2 n+1}{4 \pi} \frac{(n-|m|) !}{(n+|m|) !}} P_{n}^{|m|}\left(\widehat{x}_{3}\right) \begin{cases}\operatorname{Re}\left(\left(\widehat{x}_{1}+i \widehat{x}_{2}\right)^{m}\right), & m \geqslant 0 \\ \operatorname{Im}\left(\left(\widehat{x}_{1}+i \widehat{x}_{2}\right)^{m}\right), & m<0\end{cases}
$$

Since the spherical harmonics form an orthonormal basis of the Hilbert space of square-integrable functions defined on the unit sphere, each coordinate of the parametrization can be represented by a Fourier series which leads to the representation

$$
\boldsymbol{\gamma}(\widehat{\mathbf{x}})=\sum_{n=0}^{\infty} \sum_{m=-n}^{n} \mathbf{a}_{m, n} Y_{n}^{m}(\widehat{\mathbf{x}}), \quad \widehat{\mathbf{x}} \in \mathbb{S}
$$


with certain vector valued coefficients $\mathbf{a}_{m, n} \in \mathbb{R}^{3}$. Hence, it is reasonable to take the truncated series

$$
\boldsymbol{\gamma}_{N}(\widehat{\mathbf{x}})=\sum_{n=0}^{N} \sum_{m=-n}^{n} \mathbf{a}_{m, n} Y_{n}^{m}(\widehat{\mathbf{x}}), \quad \widehat{\mathbf{x}} \in \mathbb{S},
$$

as an approximation of $\gamma$.

Notice that the representations (3.1) and (3.2), respectively, are not unique. Indeed, if $\Xi: \mathbb{S} \rightarrow \mathbb{S}$ denotes any smooth diffeomorphism, then the composed function $\gamma_{N} \circ \Xi$ describes another parametrization of $\Gamma$. For the purpose of numerical computations, some parametrizations are preferable to others. In order to discretize functions on the free boundary, we will use in Section 3.4 the parametrization (3.2) to map a subdivision of the parameter space $\mathbb{S}$ to the actual boundary $\Gamma$. From this point of view it is obvious that, for numerical computations, a "nice" parametrization maps a uniform and regularly shaped mesh of the reference surface $\mathbb{S}$ to a uniform and regularly shaped mesh on $\Gamma$. Therefore, we will introduce in Section 3.5 a suitable mesh functional $M(\Gamma)$ to monitor the quality of the mesh and apply from time to time a remeshing step to construct an appropriate re-parametrization of the free boundary, if necessary.

\subsection{Computing the updates}

Let

$$
\boldsymbol{\gamma}_{N}^{(k)}(\widehat{\mathbf{x}})=\sum_{n=0}^{N} \sum_{m=-n}^{n} \mathbf{a}_{m, n}^{(k)} Y_{n}^{m}(\widehat{\mathbf{x}}), \quad \widehat{\mathbf{x}} \in \mathbb{S}
$$

be the parametrization of the current free boundary $\Gamma_{k}$. To compute the new parametrization, we have to project the update $\delta_{k} \mathbf{n}_{k}$ computed by either (2.5) or (2.10) into the finite representation (3.2). Since the spherical harmonics are orthonormal, we find for the update

$$
\begin{aligned}
\boldsymbol{\gamma}_{N}^{(k+1)}(\widehat{\mathbf{x}}) & =\sum_{n=0}^{N} \sum_{m=-n}^{n} \mathbf{a}_{m, n}^{(k+1)} Y_{n}^{m}(\widehat{\mathbf{x}}) \\
& =\sum_{n=0}^{N} \sum_{m=-n}^{n}\left[\mathbf{a}_{m, n}^{(k)}+\left(\delta_{k} \mathbf{n}_{k}, Y_{n}^{m}\right)_{L^{2}(\mathbb{S})}\right] Y_{n}^{m}(\widehat{\mathbf{x}}), \quad \widehat{\mathbf{x}} \in \mathbb{S} .
\end{aligned}
$$

This means that the new coefficients $\left\{\mathbf{a}_{m, n}^{(k+1)}\right\}$ are obtained as the componentwise best approximation of $\boldsymbol{\gamma}_{N}^{(k)}+\delta_{k} \mathbf{n}_{k}$ with respect to the inner product in $L^{2}(\mathbb{S})$.

\subsection{Solving the second order update equation}

The second order update $\delta_{k}^{(\mathrm{scd})}$ is given implicitly by the linear equation (2.10). To efficiently compute the sought update, we perform a Galerkin discretization of (2.10) by means of spherical harmonics: Seek $\delta_{k}=\sum_{n=0}^{N} \sum_{n=-m}^{m} b_{n}^{m} Y_{n}^{m}$ such that

$$
\begin{gathered}
(\mathbf{I}-\mathbf{A}) \mathbf{b}=\mathbf{f}, \\
\left.\left.\mathbf{A}=\left[\left(\frac{\mathrm{d} u_{k}\left[Y_{n}^{m}\right] \circ \gamma_{k}}{h \circ \gamma_{k}}, Y_{n^{\prime}}^{m^{\prime}}\right]\right)_{L^{2}(\mathbb{S})}\right]_{(n, m),\left(n^{\prime}, m^{\prime}\right)}, \quad \mathbf{f}=\left[\left(\Psi\left(\boldsymbol{\gamma}_{k}\right), Y_{n}^{m}\right]\right)_{L^{2}(\mathbb{S})}\right]_{(n, m)},
\end{gathered}
$$


where the vector $\mathbf{b}=\left[b_{n}^{m}\right]_{(n, m)}$ contains the unknown coefficients. This system of linear equations is solved by the GMRES method, introduced in [29]. In particular, in our numerical realization, we never assemble the system matrix but implement the matrix-vector product.

\subsection{Surface parametrization by quadrangular patches}

We shall assume that the boundary manifolds $\Gamma$ and $\Sigma$ are given as parametric surfaces consisting of smooth patches. More precisely, let $\square:=[0,1]^{2}$ denote the unit square. The manifold $\Gamma \cup \Sigma$ is partitioned into a finite number of patches

$$
\Gamma \cup \Sigma=\bigcup_{i=1}^{M} \Gamma_{i}, \quad \Gamma_{i}=\kappa_{i}(\square), \quad i=1,2, \ldots, M,
$$

where each $\kappa_{i}: \square \rightarrow \Gamma_{i}$ defines a diffeomorphism of $\square$ onto $\Gamma_{i}$. The intersection $\Gamma_{i} \cap \Gamma_{i^{\prime}}, i \neq i^{\prime}$, of two patches $\Gamma_{i}$ and $\Gamma_{i^{\prime}}$ is assumed to be either $\emptyset$, or a common edge, or a common vertex. A quadrangular mesh on the surface $\Gamma \cup \Sigma$ is then obtained by mapping a quadrangulation of $\square$ to $\Gamma \cup \Sigma$ via parametrization.

The construction of the parametric representation of the moving boundary $\Gamma$ should be presented in more detail. To that end, it suffices to construct such a parametrization for the reference manifold $\mathbb{S}$ which is then mapped to $\Gamma$ by $\boldsymbol{\gamma}$ : The surface of the cube $[-0.5,0.5]^{3}$ consists of six patches. Each point $\mathbf{x} \in \partial\left([-0.5,0.5]^{3}\right)$ can be lifted onto the boundary $\Gamma$ via the operation

$$
\mathbf{y}(\mathbf{x})=\gamma\left(\frac{\mathbf{x}}{\|\mathbf{x}\|}\right) \in \Gamma .
$$

In this manner, the surface $\Gamma$ is subdivided into $M=6$ patches. The parametric representations $\kappa_{i}: \square \rightarrow \Gamma_{i}$ can easily be derived from (3.4). We refer to Fig. 2 for an illustration of the proposed parametric representation and mesh generation.
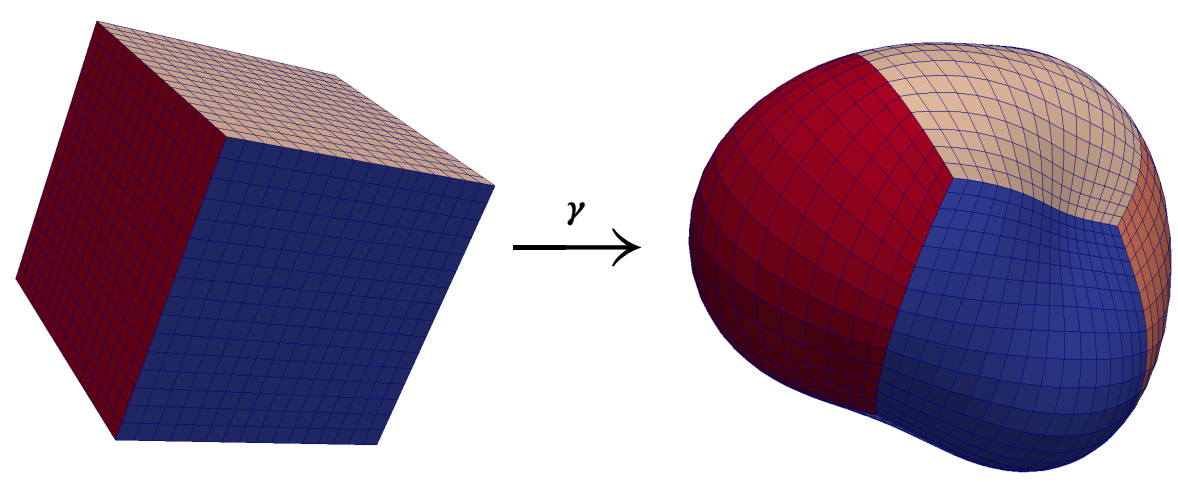

FIG. 2. Parametric representation of $\Gamma$ with quadrangular mesh on refinement level 4 


\subsection{Improvement of the mesh quality}

We shall specify the remeshing procedure as already used in [18]. A "nice" parametrization maps orthonormal tangents with respect to the unit cube onto orthogonal tangents of length $\approx|\Gamma| / 6$ with respect to the boundary $\Gamma$. This means, that the first fundamental tensor of differential geometry, given by

$$
\mathbf{K}_{i}(\mathbf{s})=\left[\left\langle\frac{\partial \boldsymbol{\kappa}_{i}}{\partial x_{j}}(\mathbf{s}), \frac{\partial \boldsymbol{\kappa}_{i}}{\partial x_{k}}(\mathbf{s})\right\rangle\right]_{j, k=1,2}, \quad \mathbf{s}=\left[s_{1}, s_{2}\right]^{T} \in \square,
$$

satisfies $\mathbf{K}_{i} \approx|\Gamma| / 6 \cdot \mathbf{I}$. Hence, the remeshing procedure will be based on the mesh functional

$$
M(\Gamma)=\sum_{i=1}^{6} \int_{\square}\left\|\mathbf{K}_{i}(\mathbf{s})-\frac{|\Gamma|}{6} \cdot \mathbf{I}\right\|_{F}^{2} \mathrm{~d} \mathbf{s} .
$$

Based on the observation that the gradient of $M(\Gamma)$ with respect to the variable $\mathbf{s}$ is tangential, we may perform several gradient steps to minimize this mesh functional to increase the quality of the mesh.

\section{Solving the state equation}

\subsection{Boundary integral equations}

We will next discuss the numerical solution of (2.1) by means of a boundary element method. To that end, for sake of convenience, we skip the iteration index $k$ in the present section. We first introduce a Newton potential $N_{f}$ satisfying

$$
-\Delta N_{f}=f \quad \text { in } \Omega \text {. }
$$

The Newton potential is assumed to be explicitly known or to be computed with sufficiently high accuracy. The latter one has to be done only once in advance of the optimization process. The underlying domain needs to be large enough to ensure that $\Omega$ stays within it. However, it can be chosen fairly simple such that fast solvers are available.

The ansatz

$$
u=N_{f}+v
$$

reduces the state equation (2.1) to a mixed problem for the Laplacian

$$
\begin{aligned}
\Delta v & =0 & & \text { in } \Omega, \\
v & =g-N_{f} & & \text { on } \Sigma, \\
-\frac{\partial v}{\partial \mathbf{n}} & =h+\frac{\partial N_{f}}{\partial \mathbf{n}} & & \text { on } \Gamma .
\end{aligned}
$$

We shall next introduce the single layer operator and the double layer operator with respect to the boundaries $\Phi, \Lambda \in\{\Gamma, \Sigma\}$ by

$$
\begin{array}{ll}
\left(V_{\Phi \Lambda} u\right)(\mathbf{x}):=\frac{1}{4 \pi} \int_{\Lambda} \frac{1}{\|\mathbf{x}-\mathbf{y}\|} u(\mathbf{y}) \mathrm{d} \sigma_{\mathbf{y}}, & \mathbf{x} \in \Phi \\
\left(K_{\Phi \Lambda} u\right)(\mathbf{x}):=\frac{1}{4 \pi} \int_{\Lambda} \frac{\left\langle\mathbf{x}-\mathbf{y}, \mathbf{n}_{\mathbf{y}}\right\rangle}{\|\mathbf{x}-\mathbf{y}\|^{3}} u(\mathbf{y}) \mathrm{d} \sigma_{\mathbf{y}}, & \mathbf{x} \in \Phi .
\end{array}
$$


Note that $V_{\Phi \Lambda}$ denotes an operator of order -1 if $\Phi=\Lambda$, i.e. $V_{\Phi \Phi}: H^{-1 / 2}(\Phi) \rightarrow H^{1 / 2}(\Phi)$, while it is an arbitrarily smoothing compact operator if $\Phi \neq \Lambda \operatorname{since} \operatorname{dist}(\Gamma, \Sigma)>0$. Likewise, if $\Sigma, \Gamma$ are $C^{2}$-smooth, the double layer operator $K_{\Phi \Phi}: L^{2}(\Phi) \rightarrow L^{2}(\Phi)$ is compact while it is arbitrarily smoothing if $\Phi \neq \Lambda$. We refer the reader to [14, 25, 33] for a detailed description of boundary integral equations.

The unknown boundary data of $v$ are determined by

$$
\left[\begin{array}{cc}
-V_{\Sigma \Sigma} & K_{\Sigma \Gamma} \\
-V_{\Gamma \Sigma} & \frac{1}{2}+K_{\Gamma \Gamma}
\end{array}\right]\left[\begin{array}{c}
\left.\frac{\partial v}{\partial \mathbf{n}}\right|_{\Sigma} \\
\left.v\right|_{\Gamma}
\end{array}\right]=\left[\begin{array}{cc}
-\left(\frac{1}{2}+K_{\Sigma \Sigma}\right) & V_{\Sigma \Gamma} \\
-K_{\Gamma \Sigma} & V_{\Gamma \Gamma}
\end{array}\right]\left[\begin{array}{c}
\left.\left(g-N_{f}\right)\right|_{\Sigma} \\
-\left.\left(h+\frac{\partial N_{f}}{\partial \mathbf{n}}\right)\right|_{\Gamma}
\end{array}\right]
$$

The boundary integral operator on the left hand side of this coupled system of boundary integral equations is continuous and satisfies a Gårding inequality with respect to $H^{-1 / 2}(\Sigma) \times L^{2}(\Gamma)$. Since its injectivity follows from potential theory, this system of integral equations is uniquely solvable according to the Riesz-Schauder theory.

Likewise to (4.4), the unknown boundary data of the local shape derivative $\mathrm{d} u=\mathrm{d} u[\delta]$, defined in (2.7), are derived by the boundary integral equation

$$
\begin{aligned}
& {\left[\begin{array}{cc}
-V_{\Sigma \Sigma} & K_{\Sigma \Gamma} \\
-V_{\Gamma \Sigma} & \frac{1}{2}+K_{\Gamma \Gamma}
\end{array}\right]\left[\begin{array}{l}
\left.\frac{\partial \mathrm{d} u}{\partial \mathbf{n}}\right|_{\Sigma} \\
\left.\mathrm{d} u\right|_{\Gamma}
\end{array}\right]} \\
& =\left[\begin{array}{cc}
-\left(\frac{1}{2}+K_{\Sigma \Sigma}\right) & V_{\Sigma \Gamma} \\
-K_{\Gamma \Sigma} & V_{\Gamma \Gamma}
\end{array}\right]\left[\left.\left\{\delta\left[f-2 \mathcal{H}-\frac{\partial h}{\partial \mathbf{n}}\right]+\operatorname{div}_{\Gamma}\left(\delta \nabla_{\Gamma} u\right)\right\}\right|_{\Gamma}\right] .
\end{aligned}
$$

We would like to point out that the boundary integral operators are identical to those in (4.4) but the input data are different.

\subsection{Wavelet based boundary element methods}

We shall introduce the wavelet Galerkin method to solve the boundary integral equations (4.4) and (4.5). As a crucial ingredient, we need a hierarchy of trial spaces $V_{j} \subset V_{j+1} \subset L^{2}(\partial \Omega)$. Such spaces can be constructed employing the parametric representation of the boundary.

We introduce a mesh of level $j$ on the unit square by dyadic subdivisions of depth $j$ into $4^{j}$ squares. On this mesh we consider piecewise bilinear nodal basis functions $\left\{\varphi_{j, k}^{\square}: k \in \triangle_{j}^{\square}\right\}$, where $\triangle_{j}^{\square}$ denotes a suitable index set satisfying $\left|\triangle_{j}^{\square}\right|=\left(2^{j}+1\right)^{2}$. We define a set of basis functions on the surface $\Gamma$ via parametrization

$$
\varphi_{j, k}^{\Gamma_{i}}(\mathbf{x}):= \begin{cases}\varphi_{j, k}^{\square}(\mathbf{s}), & \mathbf{x}=\gamma_{i}(\mathbf{s}) \in \Gamma_{i}, \\ 0, & \text { elsewhere }\end{cases}
$$

where $i=1,2, \ldots, M$. Glueing along the interfaces of the patches yields continuous bilinear ansatz functions $\left\{\varphi_{j, k}^{\partial \Omega}: k \in \triangle_{j}\right\}$, where $\left|\triangle_{j}\right| \sim M \cdot 4^{j}$. Obviously, the trial spaces

$$
V_{j}:=\operatorname{span}\left\{\varphi_{j, k}^{\partial \Omega}: k \in \triangle_{j}\right\} \subset H^{1}(\partial \Omega)
$$

are nested with respect to $j$.

To obtain the Galerkin formulation of (4.4), we introduce for $\Phi, \Lambda \in\{\Sigma, \Gamma\}$ the system matrices

$$
\mathbf{V}_{\Phi \Lambda}=\left[\left(V_{\Phi \Lambda} \varphi_{j, k^{\prime}}^{\Lambda}, \varphi_{j, k}^{\Phi}\right)_{L^{2}(\Phi)}\right]_{k, k^{\prime}}, \quad \mathbf{K}_{\Phi \Lambda}=\left[\left(K_{\Phi \Lambda} \varphi_{j, k^{\prime}}^{\Lambda}, \varphi_{j, k}^{\Phi}\right)_{L^{2}(\Phi)}\right]_{k, k^{\prime}}
$$


the mass matrices

$$
\mathbf{G}_{\Phi}=\left[\left(\varphi_{j, k^{\prime}}^{\Phi}, \varphi_{j, k}^{\Phi}\right)_{L^{2}(\Phi)}\right]_{k, k^{\prime}}
$$

and the load vectors

$$
\mathbf{f}_{\Sigma}=\left[\left(g-N_{f}, \varphi_{j, k}^{\Sigma}\right)_{L^{2}(\Sigma)}\right]_{k}, \quad \mathbf{f}_{\Gamma}=\left[-\left(h+\frac{\partial N_{f}}{\partial \mathbf{n}}, \varphi_{j, k}^{\Gamma}\right)_{L^{2}(\Gamma)}\right]_{k} .
$$

This leads to the boundary element method

$$
\left[\begin{array}{cc}
-\mathbf{V}_{\Sigma \Sigma} & \mathbf{K}_{\Sigma \Gamma} \\
-\mathbf{V}_{\Gamma \Sigma} & \frac{1}{2} \mathbf{G}_{\Gamma}+\mathbf{K}_{\Gamma \Gamma}
\end{array}\right]\left[\begin{array}{c}
\mathbf{v}_{\Sigma} \\
\mathbf{v}_{\Gamma}
\end{array}\right]=\left[\begin{array}{cc}
-\left(\frac{1}{2} \mathbf{G}_{\Sigma}+\mathbf{K}_{\Sigma \Sigma}\right) & \mathbf{V}_{\Sigma \Gamma} \\
-\mathbf{K}_{\Gamma \Sigma} & \mathbf{V}_{\Gamma \Gamma}
\end{array}\right]\left[\begin{array}{l}
\mathbf{G}_{\Sigma}^{-1} \mathbf{f}_{\Sigma} \\
\mathbf{G}_{\Gamma}^{-1} \mathbf{f}_{\Gamma}
\end{array}\right]
$$

which approximates the Dirichlet data $\left.v\right|_{\Gamma} \approx \sum_{k}\left[\mathbf{v}_{\Gamma}\right]_{k} \varphi_{j, k}^{\Gamma}$ at the free boundary $\Gamma$ and the Neumann data $\left.(\partial v / \partial \mathbf{n})\right|_{\Gamma} \approx \sum_{k}\left[\mathbf{v}_{\Sigma}\right]_{k} \varphi_{j, k}^{\Sigma}$ at the interior boundary $\Sigma$. The sought Dirichlet and Neumann boundary data of the local shape derivative are computed in complete analogy where, by applying integration by parts, the load vector is given by

$$
\mathbf{f}_{\Sigma}=\mathbf{0}, \quad \mathbf{f}_{\Gamma}=\left[\left(\delta\left[f-2 \mathcal{H}-\frac{\partial h}{\partial \mathbf{n}}\right], \varphi_{j, k}^{\Gamma}\right)_{L^{2}(\Gamma)}-\left(\delta \nabla_{\Gamma} u, \nabla_{\Gamma} \varphi_{j, k}^{\Gamma}\right)_{L^{2}(\Gamma)}\right]_{k} .
$$

Unfortunately, the system matrices $\mathbf{V}_{\Phi \Lambda}$ and $\mathbf{K}_{\Phi \Lambda}$ in (4.6) are densely populated. Moreover, the system matrix $\mathbf{V}_{\Phi \Phi}$ becomes more and more ill-conditioned when the level $j$ of resolution increases. Thus, we end up with an at least quadratic complexity for computing the approximate solution of (4.3), i.e., the computational work scales at least like $\mathcal{O}\left(\left|\triangle_{j}\right|^{2}\right)$.

Instead of the single-scale basis $\left\{\varphi_{j, k}^{\partial \Omega}: k \in \triangle_{j}\right\}$, we will employ appropriate biorthogonal spline wavelets for the discretization of the boundary integral operators. Then, we obtain quasisparse system matrices having only $\mathcal{O}\left(\left|\triangle_{j}\right|\right)$ relevant matrix coefficients. Moreover, due to the norm equivalences of wavelet bases, an appropriate scaling of the bases yields a well-conditioned system of linear equations (cf. [7]). Applying the matrix compression strategy developed in [6] combined with an exponentially convergent $h p$-quadrature method [17], the wavelet Galerkin method produces the approximate solution of (4.3) within underlying discretization error accuracy in linear complexity.

\section{Numerical results}

\subsection{First example}

We will present numerical results which demonstrate the feasibility and efficiency of the second order convergent trial method that has been introduced in the previous sections. For our first example, let the interior domain $S$ be the L-shape

$$
S=\left([-0.5,0.5] \times[-1,1]^{2}\right) \backslash\left([-0.5,0.5] \times[0,1]^{2}\right)
$$

and consider the following free boundary problem: Find the free boundary $\Gamma$ with associated solution $u=u(\Gamma)$ such that it holds

$$
\begin{array}{rr}
-\Delta u=1 & \text { in } \Omega, \\
u=1 & \text { on } \Sigma, \\
-\frac{\partial u}{\partial \mathbf{n}}=h, \quad u=0 & \text { on } \Gamma .
\end{array}
$$


The constant field $h$ will vary in the example according to $h \equiv 1,2,3,4,5$. Notice that this example coincides with that from [10, 15], where first and second order shape optimization algorithms have been used for its solution.

For the problem under consideration, the inexact Newton update (2.10) coincides with the Newton update (2.9) since $h$ is constant and thus $\partial h / \partial \mathbf{n} \equiv 0$. Hence, the second order convergent trial method constitutes the Newton method. In addition to the results of the second order convergent trial method, we shall also present results of the first order convergent trial method which uses the update (2.5), multiplied by the damping factor 0.1 to enforce convergence. It corresponds to the standard method, cf. [12,36] and the references therein, and has been applied for sake of comparison.

The surface of the L-shape is parametrized via 10 patches which yields, together with the 6 patches for the representation of the free boundary, 16 surface patches in all. The state equation is then discretized on refinement level 4 which yields about 4500 piecewise bilinear boundary elements, cf. Section 4 . The particular Newton potential we use is analytically given by $N_{f}(x, y, z)=-\left(x^{2}+y^{2}+z^{2}\right) / 6$. To approximate the free boundary, we use the first 100 spherical harmonics per coordinate of the parametrization, that is, harmonic polynomials up to order $N=10$ can be represented exactly. In accordance with (3.2), this yields 300 design parameters in all. Furthermore, we apply 400 spherical harmonics for the Galerkin scheme (3.3) to compute the inexact Newton update.

In all calculations, the sphere of radius 4 has been used as an initial guess for the free boundary. The computed free boundaries and the underlying mesh are presented in Figure 3 for $h=2$ and in Figure 4 for $h=4$. The results are in good agreement with those presented in [10, 15]. Figure 5 shows in addition all the converged boundaries for $h=1,2,3,4,5$. Here, it is clearly seen that the free boundary approaches the interior L-shape as $h$ increases. Note that the mesh quality was always good during the iterative solution procedure for both trial methods such that remeshing has never been necessary.

As seen in Figure 6, the first order convergent trial method converges very slowly while the second order convergent trial method needs at most 8 iterations to produce updates of the free boundary whose norm is always less than $10^{-3}$. For the latter one, there are required in addition $8-$ 10 iterations of the GMRES method per step to solve the update equation (2.10). This means that at most 10 times the local shape derivative (2.7) has to be determined. Nevertheless, its determination is cheap compared to the assembling of the system (4.6) of linear equations which has to be performed once per iteration step of both trial methods. In that sense, the speed-up of the second order convergent trial method is essentially proportional to the reduction of the number of iterations of the trial method.

All computations have been carried out on a single processor of a computing server with 12 Intel(R) Xeon(R) E5-2643 CPUs with a clock rate of $2.6 \mathrm{GHz}$ and $256 \mathrm{~GB}$ main memory. The algorithm needs about 40 seconds per iteration step. Hence, the free surface computation by the second order convergent trial method consumes about five minutes computing time in all.

\subsection{Second example}

For our second example, let the interior domain $S$ be the U-shape

$$
S=([-0.5,0.5] \times[-1.5,1.5] \times[-1,1]) \backslash\left([-0.5,0.5] \times[-0.5,0.5]^{2}\right) \text {, }
$$



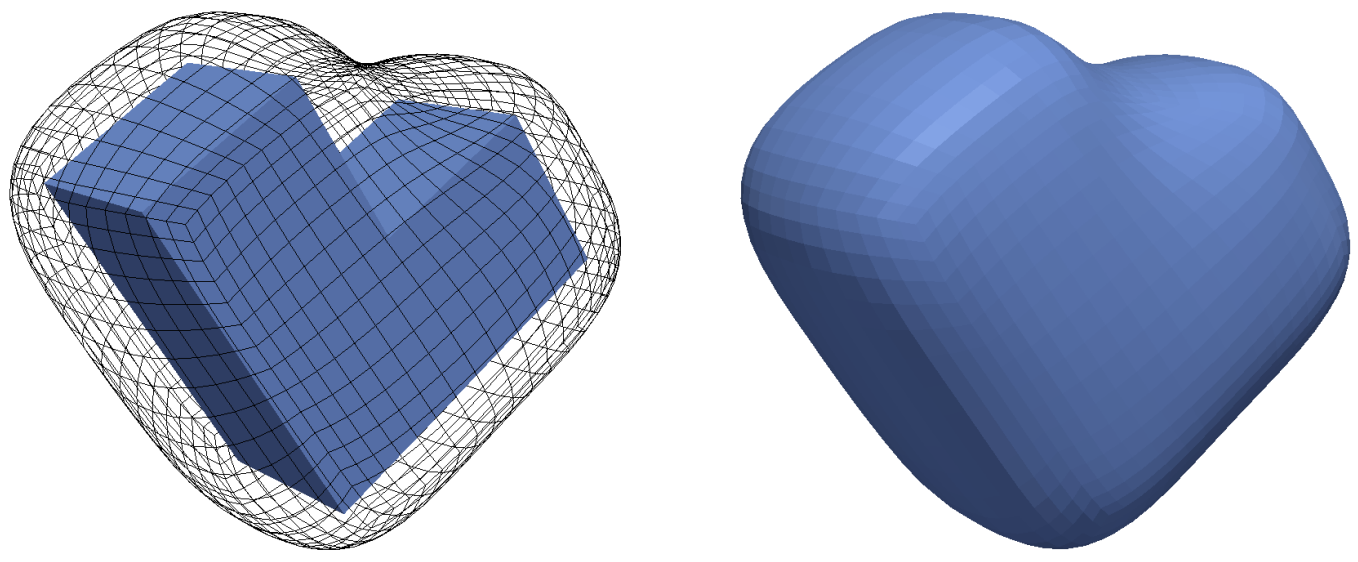

FIG. 3. The free boundary of the first example in case of $h=2$
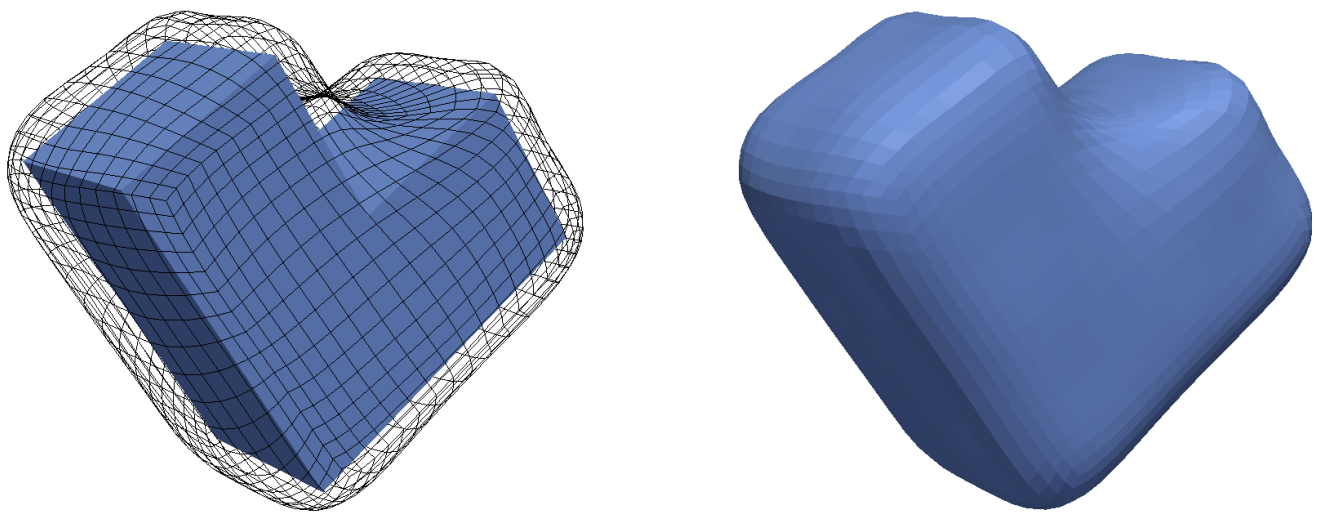

FIG. 4. The free boundary of the first example in case of $h=4$

whose surface is represented via 14 patches. We consider Bernoulli's original free boundary problem, i.e., we seek the free boundary $\Gamma$ with associated solution $u=u(\Gamma)$ such that it holds

$$
\begin{aligned}
\Delta u=0 & & \text { in } \Omega, \\
u=1 & & \text { on } \Sigma, \\
-\frac{\partial u}{\partial \mathbf{n}}=h, \quad u=0 & & \text { on } \Gamma .
\end{aligned}
$$

The field $h$ is again constant and varies according to $h \equiv 1,2,3$. Consequently, the inexact Newton update used in the second order convergent trial method coincides again with the Newton update.

To resolve the free boundary sufficiently accurate, we need to use Fourier series of length 400 for the representation of each coordinate of the parametrization, that is, harmonic polynomials up to 


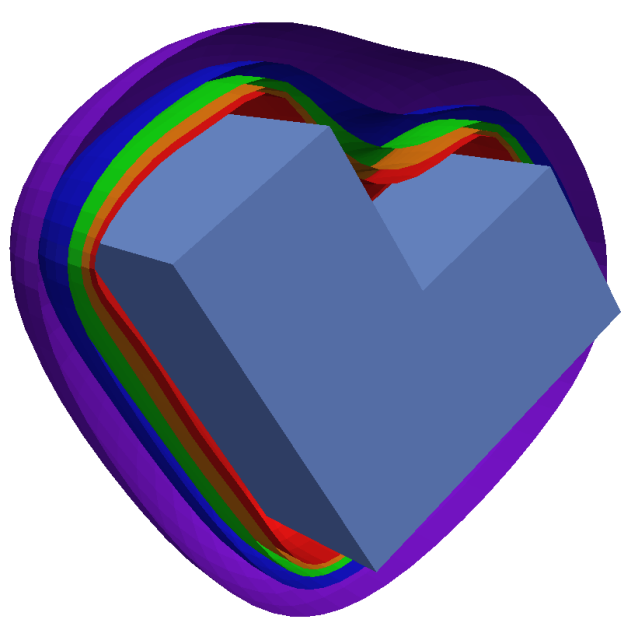

FIG. 5. The boundaries of the first example in case of $h=1,2,3,4,5$ (from outside to inside)

order 20 can be represented exactly. Hence, we have 1200 design parameters in all. When using only Fourier series of length 100 in each coordinate like in the first example, we have not been able to resolve the geometric details of the free boundary in case of $h=3$, see also Figure 8. The increase of accuracy in the boundary representation requires also an increase in the level of resolution in the discretization of the boundary integral equation. Thus, on level 5, we have about 22000 piecewise bilinear boundary elements to discretize the domain's surface. For the Galerkin scheme (3.3), we apply 1600 spherical harmonics. These settings result in a computing time of about 10 minutes per iteration of the first or second order convergent trial method on a node with Intel(R) Xeon(R) E5-2670 CPU at a clock rate of $2.6 \mathrm{GHz}$ and $256 \mathrm{~GB}$ main memory.

The results of our simulation are shown in Figure 7 for $h=1$ and in Figure 8 for $h=3$. For comparison reasons, in Figure 9, the cross sections through the converged free boundaries for
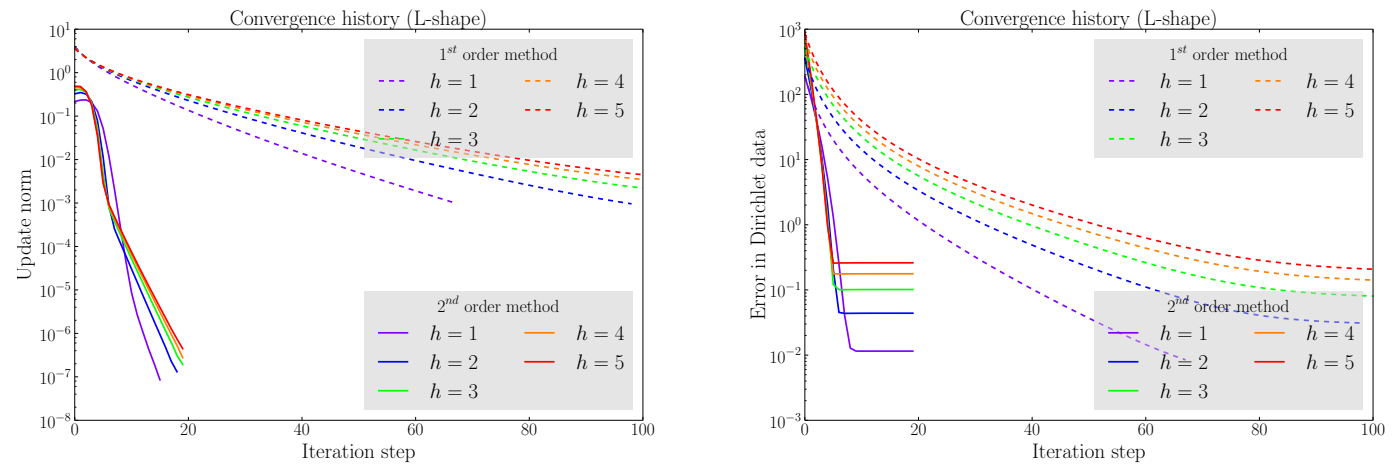

FIG. 6. Histories of the norm of the update (left) and of the mismatch of the desired Dirichlet datum (right) in case of the first example 

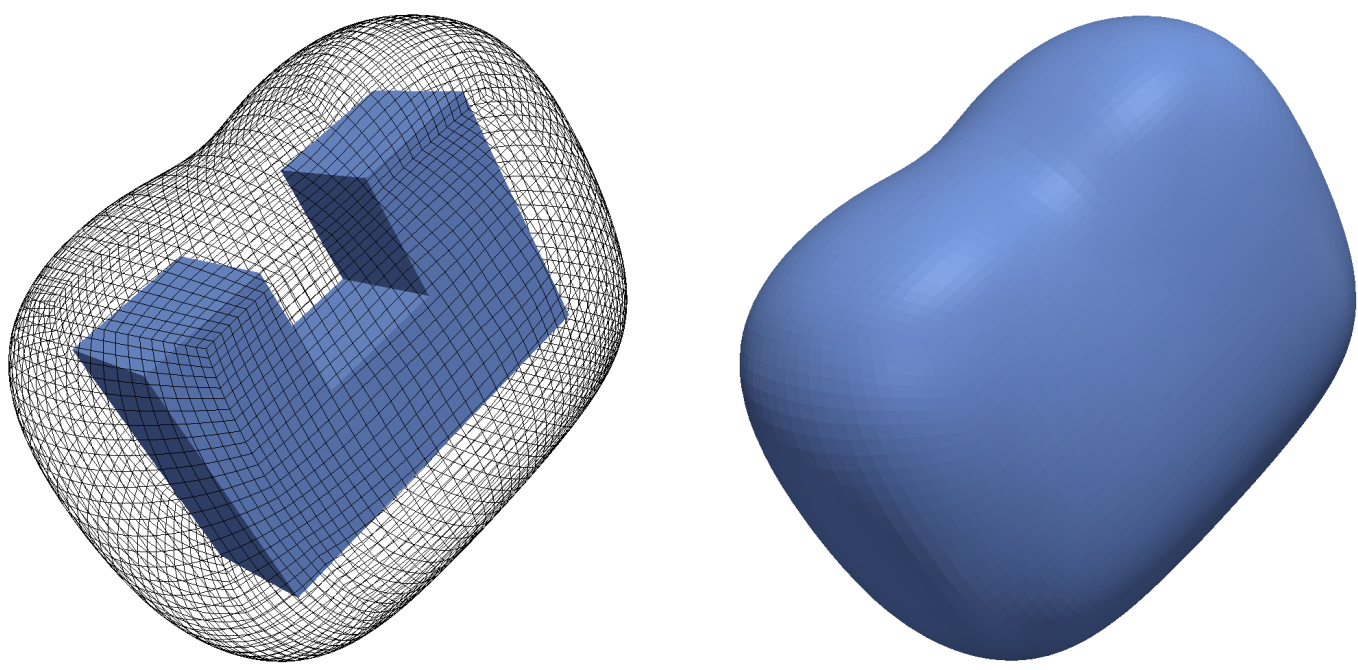

FIG. 7. The free boundary of the second example in case of $h=1$
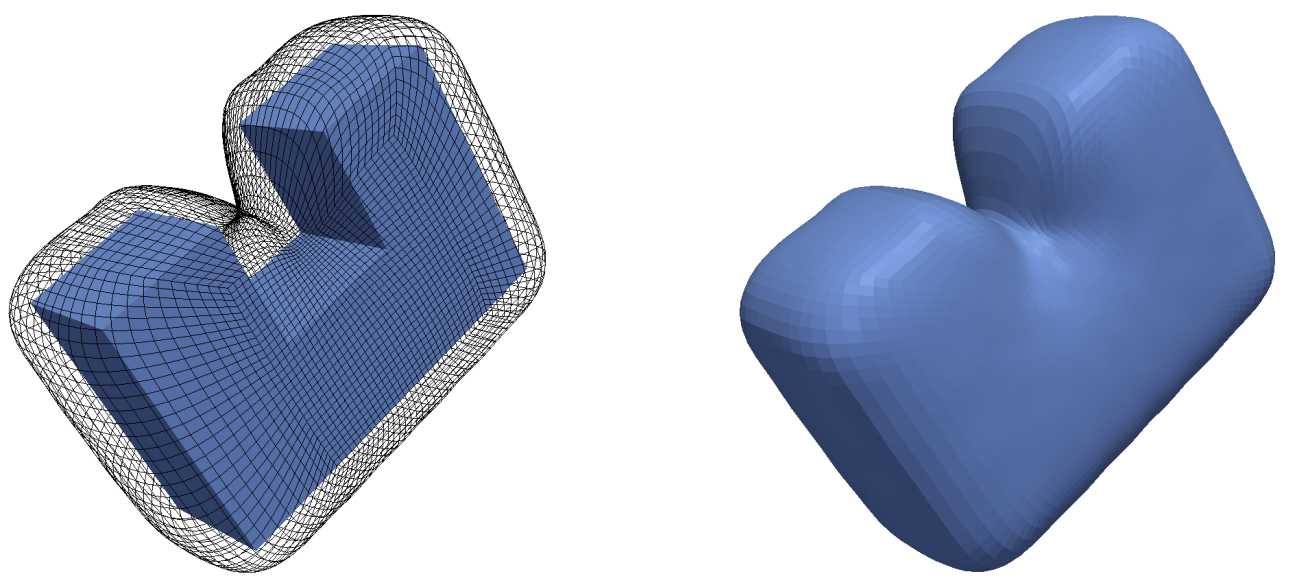

FIG. 8. The free boundary of the second example in case of $h=3$

$h=1,2,3$ are presented. The mesh quality was always very good during the iteration steps of the first and second order convergent trial method such that there was again no need for remeshing. Also, same as before, the second order convergent trial method converges much faster than the first order convergent trial method, as seen in Figure 10, where the histories of the norm of the update and the mismatch to the desired homogeneous Dirichlet datum are plotted. We observe that the second order convergent trial method requires only about 5 iterations of about 10 minutes to have updates whose norms are always less than $10^{-3}$. Compared to this, the first order convergent trial 


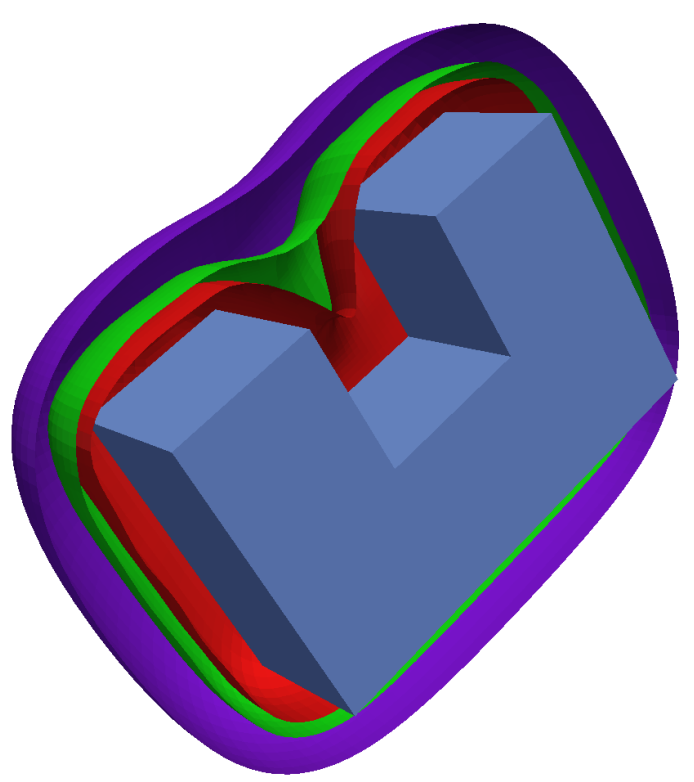

FIG. 9. The boundaries of the second example in case of $h=1,2,3$ (from outside to inside)
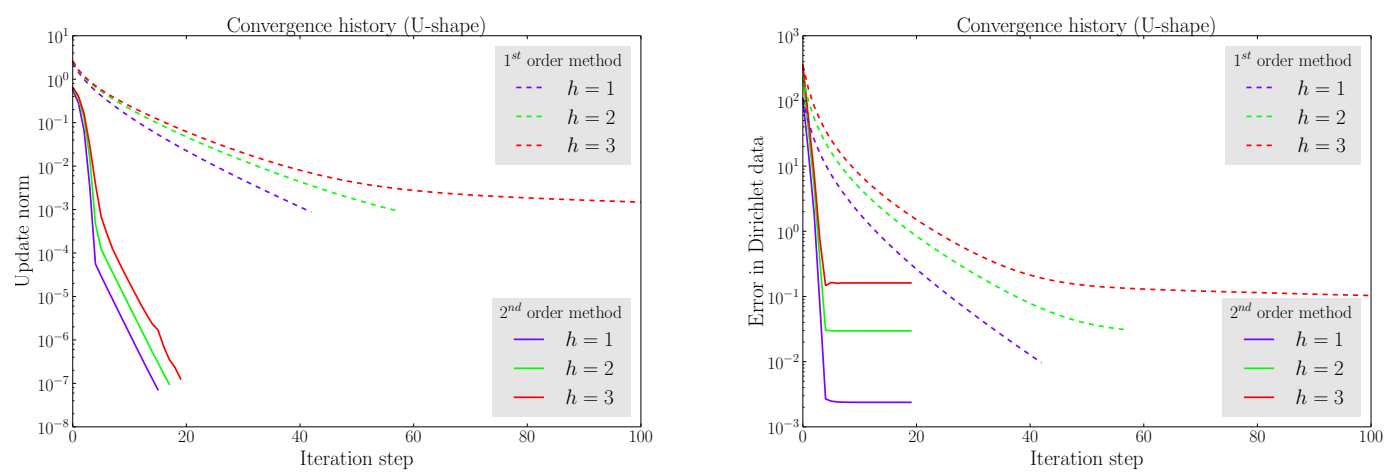

FIG. 10. Histories of the norm of the update (left) and of the mismatch of the desired Dirichlet datum (right) in case of the second example

method requires about 10 times as many iterations. Hence, the use of the second order convergent trial method results in a speed-up by a factor of about ten, which means less than 1 hour computing time versus more than 10 hours computing time.

\subsection{Third example}

By the last example presented here, we intend to demonstrate that the algorithm can also be applied to the case of a multiply connected, interior domain. The domain is chosen as the union of four 

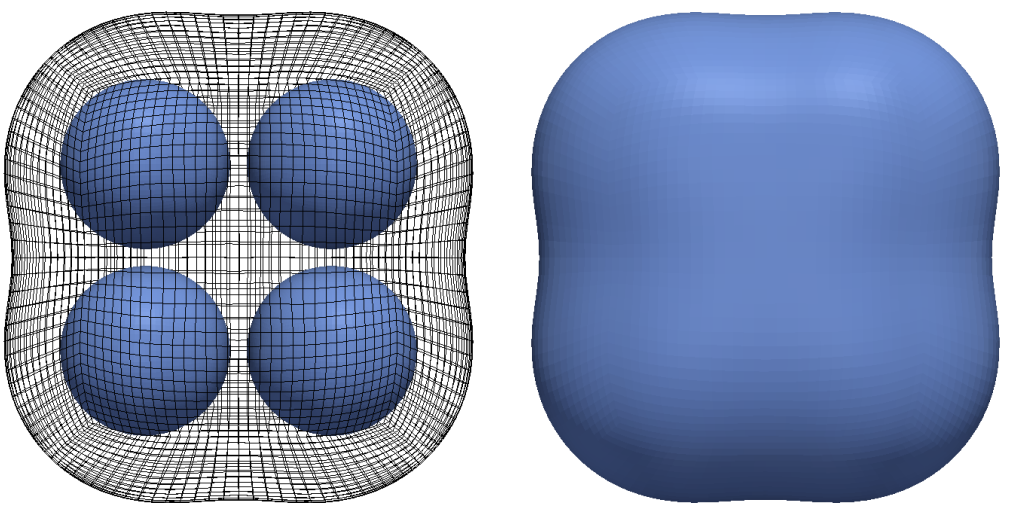

FIG. 11. The free boundary of the third example in case of $h=3$

single spheres as follows:

$$
\begin{aligned}
S & =\left\{(x, y, z) \mid(x-0.5)^{2}+(y-0.5)^{2}+z^{2} \leqslant 0.5^{2}\right\} \\
& \cup\left\{(x, y, z) \mid(x+0.5)^{2}+(y-0.5)^{2}+z^{2} \leqslant 0.5^{2}\right\} \\
& \cup\left\{(x, y, z) \mid(x+0.5)^{2}+(y+0.5)^{2}+z^{2} \leqslant 0.5^{2}\right\} \\
& \cup\left\{(x, y, z) \mid(x-0.5)^{2}+(y+0.5)^{2}+z^{2} \leqslant 0.5^{2}\right\} .
\end{aligned}
$$

The problem that we are looking at is the following free boundary problem:

$$
\begin{aligned}
\Delta u & =0 & & \text { in } \Omega, \\
u & =1 & & \text { on } \Sigma, \\
-\frac{\partial u}{\partial \mathbf{n}}=h\left(1.5-\mathrm{e}^{-x^{2} / 10}\right), & u=0 & & \text { on } \Gamma .
\end{aligned}
$$

Similar to the first two examples, we will vary the intensity of the field by choosing the constant according to $h \equiv 1,2,3,4,6,8,9,10,12,14$. Since the field is not constant any more, the inexact Newton update (2.10) is indeed different from the Newton update (2.9). Thus, the second order trial method does not coincide with the Newton method in the present situation.

In the simulation, we will again use a Fourier series of length 400 as in the second example, which means that we can represent polynomials up to degree 20 exactly. The surface of each sphere is parametrized via 6 patches, which leads to 30 patches in all. The boundary integral equation was discretized on refinement level 5, resulting in about 32500 bilinear boundary element ansatz functions. The initial guess for the free boundary is always the ball with radius 3 and the damping factor for the standard update has been set to 0.05 .

The free boundaries in case of $h=3$ can be seen in Figure $11, h=6$ in Figure 12, and $h=12$ in Figure 13, respectively. Additionally, Figure 14 contains the cross sections through all the free boundaries for $h=1,2,3,4,6,8,9,10,12$ and $h=14$. There was again no need for remeshing for both, the first and the second order convergent trial method. The second order convergent trial method takes about 3-5 iterations until the norm of the update is smaller than $10^{-3}$, while the first 

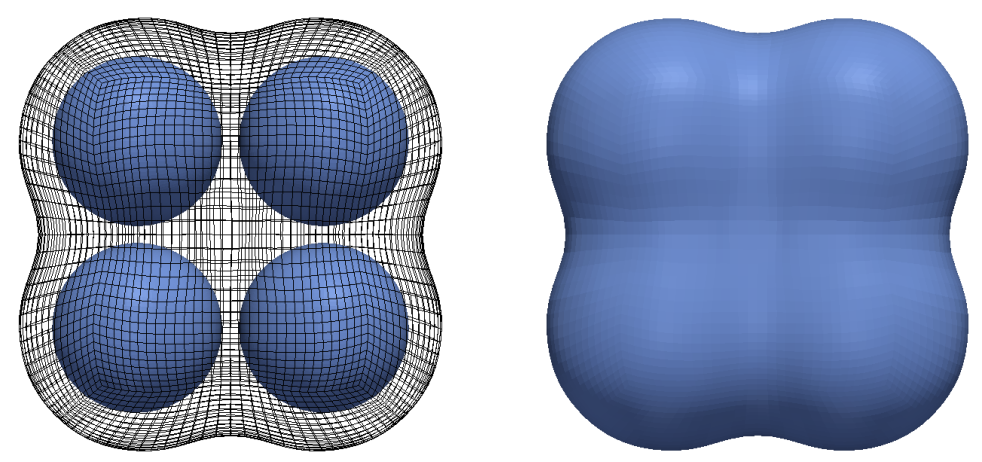

FIG. 12. The free boundary of the third example in case of $h=6$
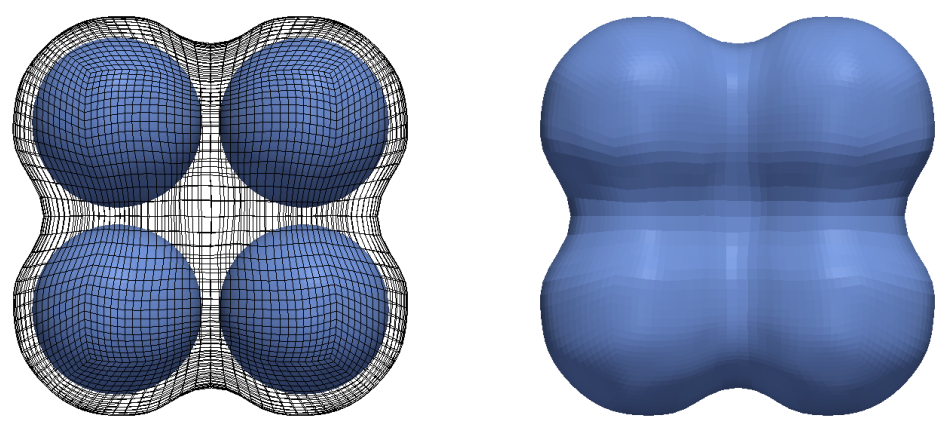

FIG. 13. The free boundary of the third example in case of $h=12$

order convergent trial method takes more than 10 times more iterations. The norm of the update and the error in the Dirichlet data at the free boundary can be seen in Figure 15. The simulation was done on the same machine as in the first example and consumed computing times of about 8 minutes per iteration for both, the first and the second order convergent trial method. Since the iteration time is basically the same, the speed-up obtained by using the second order convergent trial method is proportional to the number of iterations spared.

\section{Conclusion}

In this article, we presented a second order convergent trial method for the solution of free boundary problems. The method coincides with the Newton method if the prescribed Neumann data are constant. Otherwise, it coincides with an inexact Newton method. The method has been introduced in [16] in case of starlike domains in two spatial dimensions and boundary updates in the radial direction. Here, we provided its realization for arbitrary domains in three spatial dimensions and 


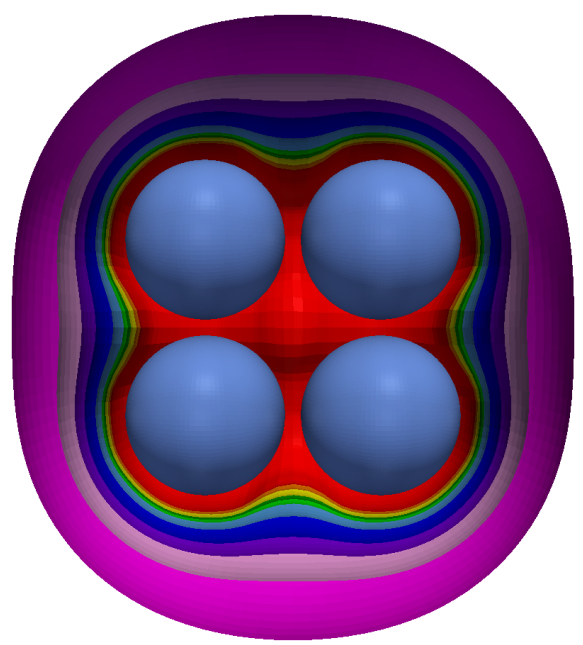

FIG. 14. The boundaries of the third example in case of $h=1,2,3,4,6,8,9,10,12$ and $h=14$ (from outside to inside)
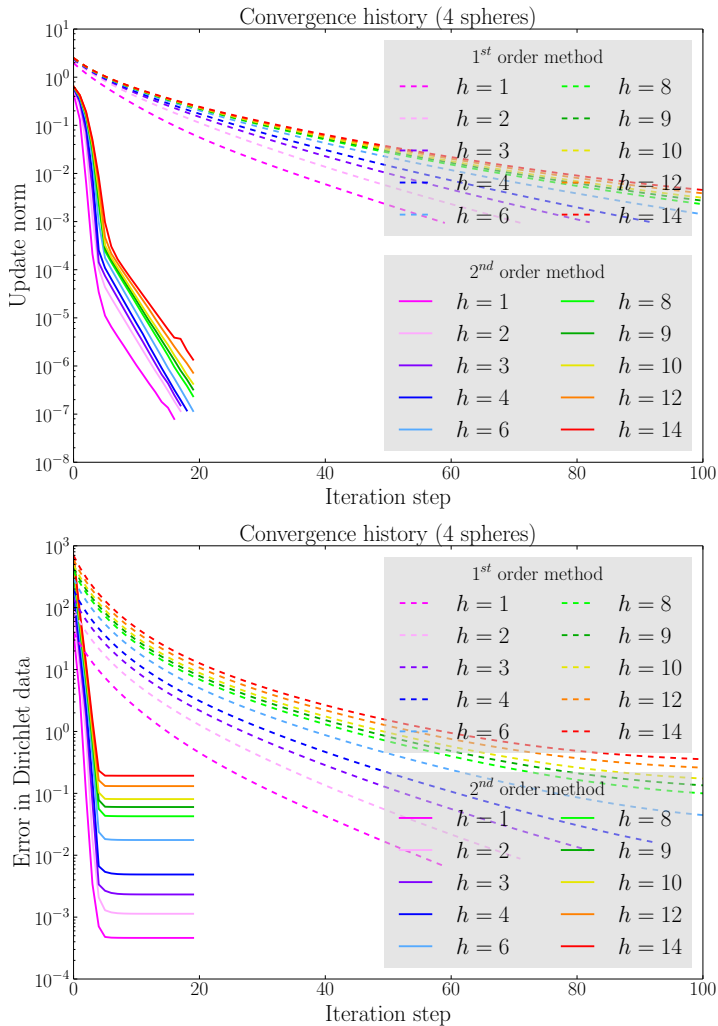

FIG. 15. Histories of the norm of the update (left) and of the mismatch of the desired Dirichlet datum (right) in case of the third example 
boundary updates in the normal direction. Numerical experiments validated the feasibility and the second order convergence of this trial method.

Acknowledgments. This research was supported by the Swiss National Science Foundation through the project "Derivative free methods in shape optimization" (grant no. 200021_137668).

\section{REFERENCES}

1. ACKER, A., How to approximate the solutions of certain free boundary problems for the Laplace equation by using the contraction principle. Z. Angew. Math. Phys. 32 (1981), 22-33. Zb10459.76009 MR0615552

2. ACKer, A., On the geometric form of Bernoulli configurations. Math. Meth. Appl. Sci. 10 (1988), 1-14. Zb10648.35085 MR0929218

3. Alt, H.W. \& CAFFARELli, L.A., Existence and regularity for a minimum problem with free boundary. J. reine angew. Math. 325 (1981), 105-144. Zb10449. 35105 MR0618549

4. Bouchon, F., Clain, S. \& Touzani, R., Numerical solution of the free boundary Bernoulli problem using a level set formulation. Comput. Methods Appl. Mech. Engrg. 194 (2005), 3934-3948. Zbl1090 . 76048 MR2149216

5. Bouchon, F., Clain, S. \& Touzani, R., A perturbation method for the numerical solution of the Bernoulli problem. J. Comput. Math. 26 (2008), 23-36. MR2378583

6. Dahmen, W., Harbrecht, H. \& Schneider, R., Compression techniques for boundary integral equations. Asymptotically optimal complexity estimates. SIAM J. Numer. Anal. 43 (2006), 2251-2271. Zbl1113.65114 MR2206435

7. Dahmen, W. \& Kunoth, A., Multilevel preconditioning. Numer. Math. 63 (1992), 315-344. Zb10757. 65031 MR1186345

8. Delfour, M. \& Zolesio, J.-P., Shapes and Geometries. Society for Industrial and Applied Mathematics (SIAM), Philadelphia, PA, 2001. Zbl1002.49029 MR1855817

9. Eppler, K. \& HARBrecht, H., On a Kohn-Vogelius like formulation of free boundary problems. Comput. Optim. Appl. 52 (2012), 69-85. Zbl1258. 49069 MR2925765

10. Eppler, K. \& Harbrecht, H., Tracking Neumann data for stationary free boundary problems. SIAM J. Control Optim. 48 (2009), 2901-2916. Zbl1202 . 49052 MR2559101

11. Eppler, K. \& HARBRECht, H., Shape optimization for free boundary problems. Analysis and numerics. In G. Leugering et al., editors, Constrained Optimization and Optimal Control for Partial Differential Equations, vol. 160 of Internat. Ser. Numer. Math., pp. 277-288, Birkhäuser, Basel, 2012. MR3060479

12. FluCher, M. \& RUMPF, M., Bernoulli's free-boundary problem, qualitative theory and numerical approximation. J. reine angew. Math. 486 (1997), 165-204. Zb10909. 35154 MR1450755

13. Geiger, C. \& Kanzow, C., Theorie und Numerik restringierter Optimierungsaufgaben. Springer, Berlin-Heidelberg, 2002. Zbl1003.90044

14. HACKBUSCH, W., Integralgleichungen. B.G. Teubner, Stuttgart, 1989. Zb10681.65099 MR1010893

15. HARBRECht, H., A Newton method for Bernoulli's free boundary problem in three dimensions. Computing, 82:11-30, 2008. Zbl1152.49044 MR2395266

16. Harbrecht, H. \& Mitrou, G., Improved trial methods for a class of generalized Bernoulli problems. J. Math. Anal. Appl. 420 (2014), 177-194. Zbl1292.35341 MR3229818

17. HARbrecht, H. \& SChNEIDER, R., Wavelet Galerkin schemes for boundary integral equations. Implementation and quadrature. SIAM J. Sci. Comput. 27 (2006), 1347-1370. Zbl1117.65162 MR2199752

18. Harbrecht, H. \& TAUSCH, J., On the numerical solution of a shape optimization problem for the heat equation. SIAM J. Sci. Comput. 35 (2013), A104-A121. Zb11264. 65156 MR3033039

19. Haslinger, J., Ito, K., Kozubek, T., Kunisch, K. \& Peichl, G., On the shape derivative for problems of Bernoulli type. Interfaces Free Bound. 11 (2009), 317-330. Zbl1178.49055 MR2511644 
20. Haslinger, J., K., Kozubek, T., Kunisch, K. \& Peichl, G., Shape optimization and fictitious domain approach for solving free boundary problems of Bernoulli type. Comput. Optim. Appl. 26 (2003), 231-251. Zbl1077.49030 MR2013364

21. Haslinger, J., Kozubek, T., Kunisch, K. \& Peichl, G., An embedding domain approach for a class of 2-d shape optimization problems: mathematical analysis. J. Math. Anal. Appl. 290 (2004), 665-685. Zb11034.49042 MR2033050

22. Haslinger, J. \& MäKInen, R.A.E., Introduction to Shape Optimization. Theory, Approximation, and Computation. Society for Industrial and Applied Mathematics (SIAM), Philadelphia, PA, 2003. Zbl1020 . 74001 MR1969772

23. Hörmander, L., The Analysis of Linear Partial Differential Operators. Springer, New York, 198385. Zbl0521. 35001 (Vol. I, 1983), Zbl0521.35002 (Vol. II, 1983) Zbl0601.35001 (Vol. III, 1985), Zbl0612. 35001 (Vol. IV, 1985) MR0717035 (Vol. I, 1983), MR0705278 (Vol. II, 1983) MR0781536 (Vol. III, 1985), MR0781537 (Vol. IV, 1985)

24. Ito, K., KUnisch, K. \& PeIChL, G., Variational approach to shape derivatives for a class of Bernoulli problems. J. Math. Anal. Appl. 314 (2006), 126-149. Zbl1088. 49028 MR2183542

25. KRESS, R., Linear Integral Equations. Springer, Berlin-Heidelberg, 1989. Zb10671. 45001 MR1007594

26. Kuster, C. M., Gremaud, P. A. \& Touzani, R., Fast numerical methods for Bernoulli free boundary problems. SIAM J. Sci. Comput. 29 (2007), 622-634. Zb11136. 65113 MR2306261

27. Murat, F. \& Simon, J., Étude de problèmes d'optimal design. In Optimization Techniques, Modeling and Optimization in the Service of Man, edited by J. Céa, Lect. Notes Comput. Sci. 41, pp. 54-62, Springer, Berlin, 1976. Zbl0334.49013

28. Pironneau, O., Optimal Shape Design for Elliptic Systems. Springer, New York, 1984. Zb10534. 49001 MR0725856

29. SAAD, Y. \& SChUltZ, M.H., GMRES: A generalized minimal residual algorithm for solving nonsymmetric linear systems. SIAM J. Sci. and Stat. Comput. 7 (1986), 856-869. Zb10599.65018 MR0848568

30. SACKinger, P. A., Schunk, P. R. \& RaO, R. R., A Newton-Raphson pseudo-solid domain mapping technique for free and moving boundary problems. A finite element implementation. J. Comput. Phys. 125 (1996), 83-103. Zb10853.65138 MR1381805

31. Simon, J., Differentiation with respect to the domain in boundary value problems. Numer. Funct. Anal. Optim. 2 (1980), 649-687. Zbl0471. 35077 MR0619172

32. Sokolowski, J. \& Zolesio, J.-P., Introduction to Shape Optimization. Springer, Berlin, 1992. Zb10761.73003 MR1215733

33. SteInbach, O., Numerical Approximation Methods for Elliptic Boundary Value Problems. Finite and Boundary Elements. Springer, New York, 2008. Zb11153.65302 MR2361676

34. TEPPER, D.E., On a free boundary value problem, the starlike case. SIAM J. Math. Anal. 6 (1975), 503-505. Zbl0299.31004 MR0367237

35. TilHonen, T., Shape optimization and trial methods for free-boundary problems. RAIRO Model. Math. Anal. Numér. 31 (1997), 805-825. Zb10891.65131 MR1489173

36. Tilhonen, T. \& JÄrvinen, J., On fixed point (trial) methods for free boundary problems. In S.N. Antontsev et al., editors, Free Boundary Problems in Continuum Mechanics (Novosibirsk, 1991), vol. 106 of Internat. Ser. Numer. Math., pp. 339-350, Birkhäuser, Basel, 1992. Zb10817.35135 MR1229552

37. Toivanen, J.I., Haslinger, J. \& Mäkinen, R. A.E., Shape optimization of systems governed by Bernoulli free boundary problems. Comput. Methods Appl. Mech. Engrg., 197:3803-3815, 2008. Zb11194.76031 MR2458115

38. Zolesio, J.-P., Numerical algorithms and existence result for a Bernoulli-like steady free boundary problem. Large Scale Systems 6 (1984), 263-278. Zbl0552 . 49027 MR0780683 